\title{
Capillary network formation from dispersed endothelial cells: Influence of cell traction, cell adhesion, and extracellular matrix rigidity
}

\author{
João R. D. Ramos, ${ }^{1,2, *}$ Rui Travasso, ${ }^{1, \dagger}$ and João Carvalho ${ }^{1, \ddagger}$ \\ ${ }^{1}$ Centro de Física da Universidade de Coimbra, CFisUC, 3007-516 Coimbra, Portugal \\ ${ }^{2}$ Max Planck Institute for Dynamics and Self-Organization, 37077 Göttingen, Germany
}

(Received 25 August 2017; revised manuscript received 11 December 2017; published 22 January 2018)

\begin{abstract}
The formation of a functional vascular network depends on biological, chemical, and physical processes being extremely well coordinated. Among them, the mechanical properties of the extracellular matrix and cell adhesion are fundamental to achieve a functional network of endothelial cells, able to fully cover a required domain. By the use of a Cellular Potts Model and Finite Element Method it is shown that there exists a range of values of endothelial traction forces, cell-cell adhesion, and matrix rigidities where the network can spontaneously be formed, and its properties are characterized. We obtain the analytical relation that the minimum traction force required for cell network formation must obey. This minimum value for the traction force is approximately independent on the considered cell number and cell-cell adhesion. We quantify how these two parameters influence the morphology of the resulting networks (size and number of meshes).
\end{abstract}

DOI: 10.1103/PhysRevE.97.012408

\section{INTRODUCTION}

The different processes that give rise to the formation of blood vessels in mammals are complex and extremely well regulated. It is hard to overstate the importance to understand these processes. In fact, the growth of new blood vessels determine the progression of more than 50 pathologies, some of which presenting a large burden in modern society [1]. For example, the inflammatory environment in the vicinity of solid tumors often promotes the growth of neovessels capable of delivering nutrients to the tumor [2,3], of completely altering the flow pattern in the tissue $[4,5]$ and, importantly, of transporting metastatic tumor cells to new locations in the body. The capacity for developing new collateral vessels, on the other hand, is associated with successful prognosis after a heart attack [6,7]. In endometriosis the development of new dense vasculatures is essential for the endometriotic lesions to survive outside the uterus [8]. In diabetic retinopathy, the growth of many neovessels, together with the presence of micro-aneurysms and hemorrhages in different locations in the retina, leads progressively to blindness [9-11]. Blood vessel growth is also pivotal in many non-disease-related processes such as the ovary cycle, wound healing, or embryo development [1,12-14].

Two main mechanisms lead to the formation of new blood vessels: angiogenesis and vasculogenesis. Angiogenesis is the growth of new blood vessels starting from an existing vasculature [2]. As an example, in sprouting angiogenesis, new blood vessels start as small sprouts from existing vessels and migrate in the direction of the cells that require nourishment [15-18]. In recent years several theoretical groups have devel-

\footnotetext{
*joao.r.d.ramos@gmail.com

†ruit@uc.pt

¥jcarlos@uc.pt
}

oped mathematical models to better understand the biological and mechanical processes underlying sprouting angiogenesis [19-21]. Vasculogenesis, on the other hand, is the process by which the initial blood vessel plexus is formed directly from the rearrangement and differentiation of the endothelial precursor cells [22]. It occurs during embryo formation [23] but is also important in wound healing [24,25]. The study of vasculogenesis permits us to better understand how cells move and are able to interact with each other to form complex structures.

When endothelial cells are set in elastic biological matrices, such as matrigel, they are able to form networks depending on the mechanical and chemical properties of these matrices [26-28]. Endothelial cells are able change their polarization and alignment to follow local mechanical cues in the matrix $[29,30]$. Though not unique to endothelial cells [31], the fact that they are able to form these networks in vitro suggests that these assays might be very informative regarding the mechanisms used by endothelial cells during vasculogenesis in vivo. In fact, the first step of vasculogenesis, and therefore capillary network formation, is the organization of endothelial cells in a network, similar to what happens in thesein vitro assays [22]. Only afterwards the cells in vivo alter their shape in order to form tubular structures capable of supporting blood flow.

Progress has been done experimentally and theoretically in the direction of understanding how endothelial cells form these networks. Several mathematical models of this process were developed, including both chemical and mechanical effects. One of the initial efforts was done by Manoussaki et al. [32,33], where the authors introduce a continuous mechanical model for the formation of these networks. According to this model, the endothelial cells exert a traction force in the matrix, which behaves viscoelastically: the local stress depends on both the strain and the strain rate. The mobility of the endothelial cells in a particular direction is hypothesized to increase 
with the strain in that direction and with the compression in the perpendicular direction. The endothelial cells exert an isotropic force proportional to their local density. Later works $[34,35]$ have obtained vascular networks resulting from the endothelial cells following the gradients of chemical factors produced by themselves. In these works, the description of the matrices mechanics is much simpler than in the previous works, and the formation of the networks hinge on the inertia term, i.e., the cells have inertia regarding the variation of their velocity. Many other works have modeled the formation of these networks [36-41], some considering as the main driving force the chemotaxis of endothelial cells-produced growth factors, others the mechanical forces exerted by endothelial cells.

Recently, several experiments on traction force microscopy shed light on the traction forces profile exerted by endothelial and tumor cells in matrices [42-45]. In wild-type cells capable of forming networks, these traction forces are typically pointed towards the cell center and are stronger at the border of the cell. The ensuing deformation of the matrix permits cell-cell communication mediated by the matrix strain [42]. This information was readily included into the Cellular-Potts Model developed by van Oers et al. [46], which implements directly the typical force field exerted by endothelial cells in two-dimensional matrices. Moreover, this computational model includes both passive forces (adhesion) and the active traction forces. Cell polarization is then influenced by the local strains produced by the traction forces. In this model the strain leads to an increase in tissue rigidity (strain-stiffening), which promotes cell polarization through durotaxis $[29,30]$. In the model, this alignment of the cell polarization with the strain is instantaneous. The dynamics observed in this cell-based model is also a function of the cells' surface tension and of the adhesion between the different cells of the system. These factors play a decisive role in regulating cell shape [47,48]. The authors have verified that there is a range of matrices' Young's moduli for which the endothelial cells are able to form networks. Nevertheless, that work has not looked into how the cell traction and the adhesion between the cells are able to condition the final network, nor it has explored thoroughly the types of networks obtained. In the present paper we will analyze in detail the model by van Oers et al., discuss under which conditions the cell networks are formed, and provide a theoretical explanation for the formations of the networks under this model.

In the next section we will briefly introduce the model by van Oers et al. In the Methods section we will describe the tools developed to classify and characterize the cell patterns observed. We then present and discuss the results of this work, where we systematically vary the matrices' Young's modulus, the cell traction force, and the cell-cell adhesion. We also vary the number of cells in the simulation and discuss the value of matrix stiffness required for network formation (which is almost independent of cell-cell adhesion and cell number). Finally, we draw the conclusions of this work.

\section{MODEL}

The model introduced in Ref. [46] describes the dynamics of a cell monolayer on top of an elastic biological substrate.
The cell migration dynamics, modeled by a Cellular Potts Model (CPM), is a function of the displacements induced by the traction forces applied by the cells on the substrate, which are calculated using a Finite Element Method (FEM). These traction forces are determined as a function of the cell shape. In this section we will present an overview of the various components of the model and how they interact with each other.

\section{A. Cellular Potts Model}

Consider a grid composed of pixels with side $L$. Each pixel is assigned a tag, $\sigma=1,2, \ldots$, which represents the cell which includes that pixel. The tag $\sigma=0$ is reserved for the pixels in the regions without cells. A cell is simply a set of pixels with the same tag, $\sigma \neq 0$. Let $\sigma_{i}$ be the tag of the $i$ th pixel. Each configuration of the system corresponds then to an energy given by the Hamiltonian

$$
\mathcal{H}=\sum_{\langle i j\rangle} J_{\sigma_{i} \sigma_{j}}\left(1-\delta_{\sigma_{i} \sigma_{j}}\right)+\sum_{\sigma \neq 0} \lambda_{\sigma}\left(\frac{V_{\sigma}-V_{t}}{V_{t}}\right)^{2},
$$

where the first term represents the energy contribution by the interfaces in the system (due to surface tension and adhesion molecules) and the second term refers to the energy cost for each cell having a volume $V_{\sigma}$ other than the equilibrium target volume, $V_{t}$. The sum over $\langle i j\rangle$ is a shorthand notation for a sum over every pairs of nearest neighbors $i$ and $j . J_{\sigma \sigma^{\prime}}$ corresponds to the energy cost of cell $\sigma$ being adjacent to cell $\sigma^{\prime}$ per pixel contact, and $\delta_{\sigma \sigma^{\prime}}$ is the Kronecker delta. Assuming all cells are of the same type and that $J_{\alpha \beta}=J_{\beta \alpha}$, there are two relevant adhesion cost parameters: the cell-matrix adhesion cost, $J_{01}$, and the cell-cell adhesion cost, $J_{11}$. The parameter $\lambda$ is the elastic energy cost per cell of changing the volume of the cell, which will be referred to as the inelasticity constant.

Other terms might be added to the Hamiltonian in order to model other phenomena, for instance, perimeter constraints, chemotaxis, and durotaxis. The last will be included in this system and will be explained in more detail below.

Cell movement will be simulated using the MetropolisHastings Algorithm (MHA), according to which only the change in energy between two configurations, $\Delta \mathcal{H}$, needs to be calculated. The MHA applied to the CPM consists of choosing a target pixel and a random source pixel from its neighborhood; a copy of the source's tag to the target pixel is attempted and corresponds to a change in energy $\Delta \mathcal{H}$; if the step is favorable $(\Delta \mathcal{H}<0)$ then the step is always accepted; otherwise, it has probability $p=e^{-\Delta \mathcal{H}}$ of being accepted.

A Monte Carlo step (MCS) is composed of a number of consecutive MHA steps equal to the number of pixels in the grid.

\section{B. Traction force model}

In this model, cells are able to apply traction forces to the ECM underneath them. After computing the forces, the mechanical response, i.e., the strain on the ECM, will be calculated using a FEM (see the Appendix). At this point we do not consider matrix remodeling or viscoelasticity. These effects are important in capillary formation, and we expect to extend the model in future studies to include them. 
The traction forces are obtained from the predictive model proposed by Lemmon and Romer [49], which rests on the assumption that each focal adhesion (FA) is pulled by every other FA belonging to the same cell with a force proportional to the distance between them. This is in accordance to the traction force profiles observed experimentally [43-45]. It is convenient to choose the locations where we calculate the forces exerted by the cells as the nodes of the FEM grid. In this way, the contribution to the force exerted applied to the pixel area around node $i$ by the FAs in node $j$ from the same cell is given by $\mu \boldsymbol{d}_{i j}$, where $\mu$ is the traction force parameter and $\boldsymbol{d}_{i j}$ is a vector from node $i$ to node $j$. Therefore, the total force applied in node $i$ is

$$
\boldsymbol{f}_{i}^{(\sigma)}=\mu \sum_{j \in \sigma} \boldsymbol{d}_{i j}
$$

Moreover, if two or more cells exert force in the same node, the resulting force is the sum of all force vectors applied in that node.

Hence, the traction forces applied to all nodes are calculated using this model after each MCS, and displacements are then computed with the FEM to serve as input for the strainstiffening model described below.

\section{Strain stiffening}

Cells are able to respond to local ECM deformations. They sense the strain and align according to the direction of higher strain. This cell-ECM coupling has been shown experimentally to rely on the nonlinear mechanical properties of the ECM [50]. Strain stiffening is a nonlinear mechanical response that a material can exhibit when being stretched when a positive strain results in an increase in stiffness along the strain direction. It is convenient to model this effect using the principal strains of a strain tensor. Here the increase in the material Young's modulus is modeled by the following expression:

$$
E(\epsilon)=E_{0} \max \left\{1,1+\frac{\epsilon}{\epsilon_{0}}\right\},
$$

where $E_{0}$ is the material Young's modulus at zero strain, $\epsilon$ is the principal strain, and $\epsilon_{0}$ is the strain-stiffening constant. This is a first order approximation. The magnitude of the strains in the system justify the use of this linear strain-stiffening approximation. In fact, when we implemented a power-law strain-stiffening dependence, according to what is observed in bundled fiber networks [51], we have found no appreciable difference in the simulated cell patterns.

This phenomenon connects the strain caused by cell traction force to the ECM mechanical changes, which will serve as an input for cell migration.

\section{Durotaxis}

During each MHA step, when the source is copied to the target, it is as if the source's cell extends in the direction $v$ of the target, and the target's cell retracts in the same direction. Furthermore, it will be assumed that each cell pixel senses the mechanical properties of the neighboring elements.
The expression used to model the difference in energy due to durotaxis is

$$
\Delta \mathcal{H}_{\mathrm{D}}= \pm f\left(E\left(\epsilon_{1}\right)\right)\left(\boldsymbol{v}_{1} \cdot \boldsymbol{v}\right)^{2} \pm f\left(E\left(\epsilon_{2}\right)\right)\left(\boldsymbol{v}_{2} \cdot \boldsymbol{v}\right)^{2},
$$

where the signs are positive for retractions and negative for extensions, and where $\epsilon_{1}$ and $\epsilon_{2}$ are the eigenvalues of the strain tensor computed at the center of the measured element, $\boldsymbol{v}_{1}$ and $\boldsymbol{v}_{2}$ are the respective eigenvectors, and $f(E)$ is a sigmoid function of the form

$$
f(E)=\frac{\alpha}{1+e^{-\beta\left(E-E_{1 / 2}\right)}}
$$

with $\alpha$ the magnitude, $\beta$ the steepness, and $E_{1 / 2}$ the Young's modulus for a magnitude of $\alpha / 2$. This model assumes that the durotaxis magnitude is proportional to FA maturation, qualitatively described by the function $f(E)$. In soft substrates FAs are unable to fully form due to being difficult to attach to the ECM. For medium ranges of $E$, FAs are able to mature, and their maturation increases linearly with stiffness until a fully matured state is reached, at very rigid substrates. For more details on the model please refer to Ref. [46].

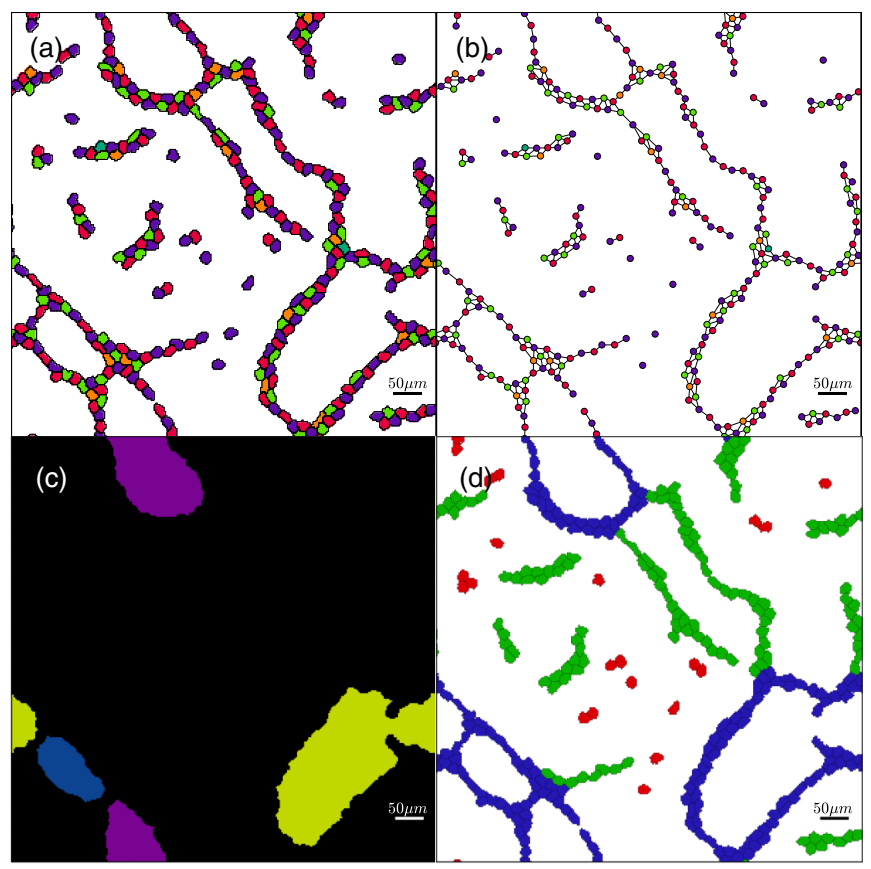

FIG. 1. The postprocessing stages of a CPM configuration of 300 cells on a $300 \times 300$ pixel $\left(750 \times 750 \mu \mathrm{m}^{2}\right)$ grid with periodic boundary conditions after 5000 MCS. (a) Resulting configuration. (b) An undirected graph representing the connections between adjacent cells. By splitting cells into different structures regarding connectivity, dispersed cells are easily found. (c) The application of a connected component algorithm to the background. Not only can cells adjacent to these regions be labeled as network cells, but one can also extract information about the number of meshes and their size. (d) Visualization of the final classification. Each cell can be dispersed (light gray), clustered (middle gray), or assembled into a network (dark gray), in color online (red, green and blue, respectively). Colors in (a), (b), and (c) simply help discern adjacent cells, nodes, and patches. 


\section{METHODS}

In order to extract information from this model regarding the morphology of the emerging cell pattern, the resulting cell configurations must be studied using image analysis techniques. Cells are classified into three distinct categories: dispersed cells, clustered cells, and cells in a network (Fig, 1).

First, cells are grouped into disjoint structures [Fig. 1(b)]: a cell belongs to a structure if it shares a boundary with an element of that structure. If the number of cells in a structure is below a certain threshold, those cells are considered dispersed. Here, if a structure has more than $n_{\text {threshold }}=4$ cells, it cannot be considered dispersed.

Next, after applying a connected component algorithm to the background pixels [Fig. 1(c)], each identified enclosed patch corresponds to a network mesh. Each cell that borders one of these patches is considered assembled into a network. In order to improve the detection of meshes, applying a few diffusion steps to the cell domains helps to close small gaps that result from the system's stochasticity. Similarly, a cutoff size for the smaller possible patch is implemented. Furthermore, from the connected component algorithm we also obtain the number and size of the network meshes. Finally, the remaining cells are regarded as clustered cells.

With this simple procedure we are able to identify approximately the cells in a network, in a cluster, and the dispersed cells. However, if there is a cluster of cells inside an enclosed patch, it will be classified as belonging to the network. Although possible, this scenario is rather unlikely

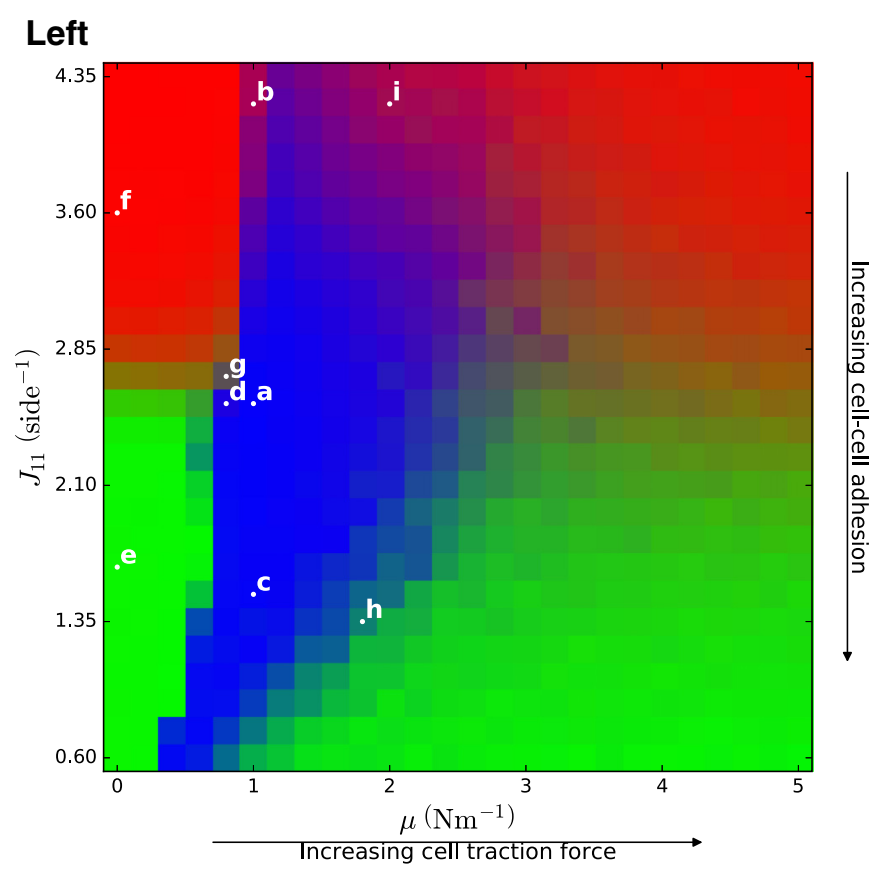

since the short-range mechanically induced alignment that is necessary for network pattern emergence disrupts the stability of clusters.

The first quantities that we extract from the simulation are the fractions of cells that belong to each of the aforementioned categories. Having used the algorithm above, this procedure is simply dividing the number of cells in a certain class by the total number of cells. To help visualize these three values, we will encode these fractions in RGB format, $\left(f_{r}, f_{g}, f_{b}\right)$, where $f_{r}$, the fraction of red, corresponds to the fraction of dispersed cells, $f_{g}$, the fraction of green, to the fraction of clustered cells, and $f_{b}$, the fraction of blue, to the fraction of cells that are organized into a network.

Other two observables are extracted from this connected component algorithm. The first one is the number of meshes, $N_{m}$, in the network, which is equal to the number of patches that are found. The second observable is the average patch size, $A_{p}$, in number of pixels. Note that, as mentioned above, patches smaller than the size of a cell are ignored.

\section{RESULTS AND DISCUSSION}

The parameters explored in this article are the cell traction force constant, $\mu$, cell-cell adhesion, $J_{11}$, and substrate stiffness, $E_{0}$. Each result reported for each set of parameters corresponds to an average of 10 simulations. The remaining parameters are the same throughout this analysis, namely, by order of appearance, $L=2.5 \mu \mathrm{m}, v=0.45, J_{01}=1.25$,

\section{Right}
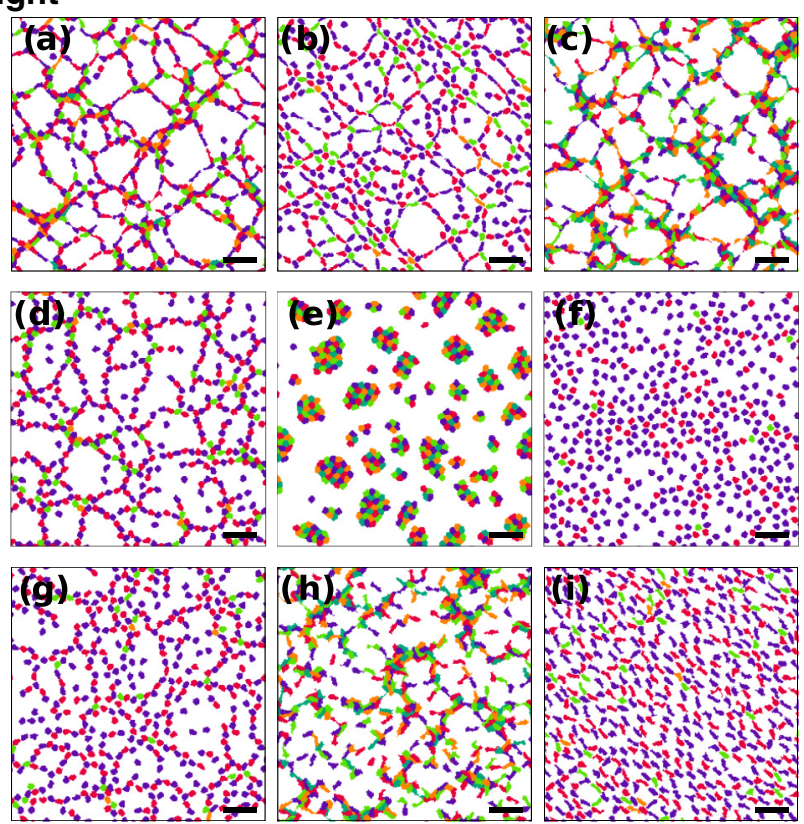

FIG. 2. (Left) RGB color-coded cell fractions, for simulations of 500 cells on a $300 \times 300$ pixel grid $\left(0.5625 \mathrm{~mm}^{2}\right)$ with rigidity $E_{0}=10$ $\mathrm{kPa}$, as a function of traction force parameter, $\mu$, from $\mu=0.0 \mathrm{Nm}^{-1}$ to $\mu=5.0 \mathrm{Nm}^{-1}$ in steps of $0.2 \mathrm{Nm}^{-1}$ and cell-cell adhesion cost, $J_{11}$, from $J_{11}=0.60$ to $J_{11}=4.35$ in steps of 0.15 . The classification categories are as follows: dispersed cells (middle gray), clustered cells (light gray), and network-like structures of cells (dark gray), in color online (red, green and blue, respectively). Therefore, the fraction of red, green, and blue in a square represents the average fraction of cells, in the aforementioned categories, over 10 independent simulations with the same values of $\mu$ and $J_{11}$, with different random number generator seeds. (Right) Chosen examples from the results presented in the left panel are shown. Scale bars: $100 \mu \mathrm{m}$. Each label from (a) through (i) represents different values of cell adhesion and cell traction force with coordinates $\left(\mu, J_{11}\right)$ : a, (1.0,2.55); b, (1.0,4.20); c, (1.0,1.50); d, (0.8,2.55); e, (0.0,1.65); f, (0.0,3.60); g, (0.8, 2.70); h, (1.8,1.35); i, (2.0,4.20). 

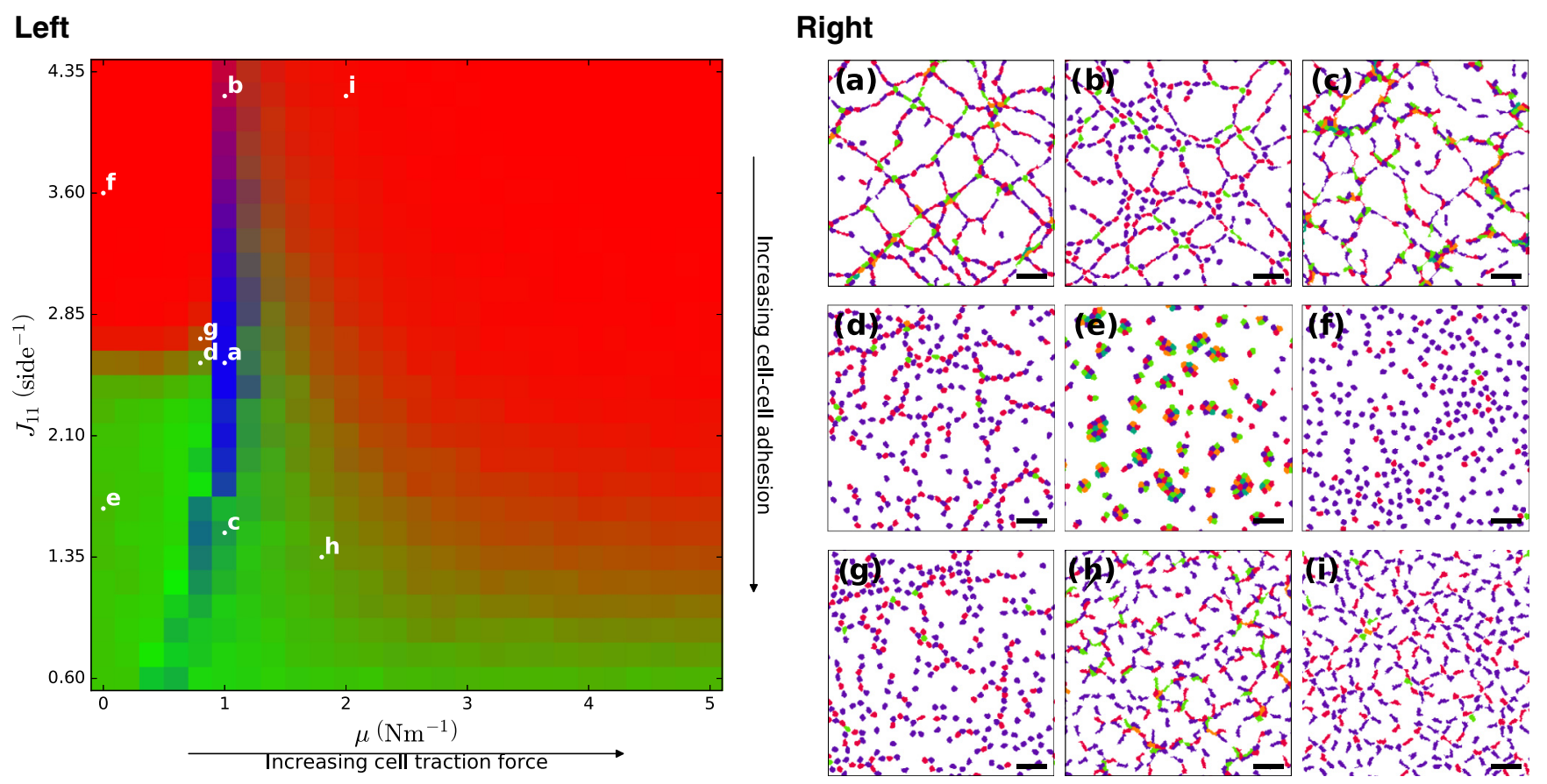

FIG. 3. (Left) RGB color-coded cell fractions, for simulations of 300 cells on a $300 \times 300$ pixel grid $\left(0.5625 \mathrm{~mm}^{2}\right)$ with rigidity $E_{0}=10$ $\mathrm{kPa}$, as a function of traction force, $\mu$, from $\mu=0.0 \mathrm{Nm}^{-1}$ to $\mu=5.0 \mathrm{Nm}^{-1}$ in steps of $0.2 \mathrm{Nm}^{-1}$ and cell-cell adhesion cost, $J_{11}$, from $J_{11}=0.60$ to $J_{11}=4.35$ in steps of 0.15 . The classification categories are as follows: dispersed cells (middle gray), clustered cells (light gray), and network-like structures of cells (dark gray), in color online (red, green and blue, respectively). Therefore, the fraction of red, green, and blue in a square represents the average fraction of cells, in the aforementioned categories, over 10 independent simulations with the same values of $\mu$ and $J_{11}$, with different random number generator seeds. (Right) Chosen examples from the results presented in the left panel are shown. Scale bars: $100 \mu \mathrm{m}$. Each label from (a) through (i) represents different values of cell adhesion and cell traction force with coordinates $\left(\mu, J_{11}\right)$ : a, (1.0,2.55); b, (1.0,4.20); c, (1.0,1.50); d, (0.8,2.55); e, (0.0,1.65); f, (0.0,3.60); g, (0.8,2.70); h, (1.8, 1.35); i, (2.0,4.20).

$\lambda=500, V_{t}=16 \pi$ pixels $=314 \mu \mathrm{m}^{2}, \epsilon_{0}=0.1, \alpha=10$, $\beta=0.5 \mathrm{kPa}^{-1}, E_{1 / 2}=15 \mathrm{kPa}$. These parameters were used to validate the model by comparing with cell cultures of bovine aortic endothelial cells [46]. Furthermore, every result is obtained on a $300 \times 300\left(750 \times 750 \mu \mathrm{m}^{2}\right)$ grid with periodic boundary conditions after $5000 \mathrm{MCS}$. After this number of iterations the observed fractions of cells dispersed, clustered, and in networks were found to stabilize.

\section{A. Classification of emerging cell structures}

Starting from a random distribution of cells, akin to in vitro assays of endothelial cells, cell structures obtained after 5000 MCS were classified into dispersed, clusters, and networks, according to the procedure previously described, for different values of traction force and cell-cell adhesion. These two parameters were chosen not only because they are dependent on different transmembrane proteins, but also because they are critical to the formation of vessel-like structures. The substrate stiffness in these initial runs was chosen to be $E_{0}=10 \mathrm{kPa}$. The parameter space was explored for $N=500$ cells (Fig. 2) and for $N=300$ cells (Fig. 3 ).

The classification as a function of cell traction force and cellcell adhesion for $N=500$ cells [Fig. 2(a)] reveals that around $\mu=0.8-1.0 \mathrm{Nm}^{-1}$ there is a transition from clusters and dispersed cells to emerging networks. With further increase of traction force the number of cell-forming networks decreases until networks cease to emerge for high values of traction force. The minimum traction force required for the formation of networks is very robust to changes in cell-cell adhesion. However, the range of possible values for traction force that allow networks to emerge decreases when deviating from medium values of cell-cell adhesion. Moreover, the value of cell-cell adhesion for which there is a transition between clusters and dispersed cells (at low traction force), $J_{11}=2.70$, is very close to the value for neutral adhesion $J_{11}=2 J_{01}=2.5$ (i.e., for when the energy of a interface between two cells is equal to the energy of the two interfaces, one from each cell, between them and the ECM).

By comparing Figs. 2 (right, a), 2 (right, b), and 2 (right, c) we observe the difference in changing cell-cell adhesion. In these figures the cells are polarized, their force field is able to deform the nearby matrix, and they are able to align along the strain. However, as the adhesion increases the cells become closer together. In Fig. 2 (right, b) the cells align in a network formation. But, because at these low adhesions it is energetically more favorable for cells to have interfaces with the matrix than with each other, the cells do not touch each other. As the adhesion increases, the cells start forming interfaces [as in Fig. 2 (right, a)] and then forming some larger groups of cells within the network [as in Fig. 2 (right, c)]. Since the number of cells is the same in these simulations, when the vessels become thicker [at higher adhesion, i.e., low $J_{11}$; see Figs. 2 (right, a) and 2 (right, c)], the meshes of the network become larger. 
When comparing Figs. 2 (right, d) and 2 (right, a) we observe that they are very different regarding cell polarization, even though the only parameter difference is a small variation in the cells' traction force. We see that between traction force values of 0.8 and $1.0 \mathrm{~N} / \mathrm{m}$ the capacity for cells to polarize increases dramatically.

Figures 2 (right, e) and 2 (right, f) show the clustered and dispersed arrangements of cells. Figure 2 (right, g) is an example of a mixed state near the transition to networks, which results from networks being highly unstable due to a lower cell-cell adhesion. Figure 2 (right, $h$ ) is an example of a intermediate state between networks and clusters in which there is a high variability in cell shape. This morphology is the result of high cell-cell adhesion and high cell traction, which strongly deforms the matrix. These two factors together imply that the shape of each cell is strongly conditioned by the position of other cells and by the forces they exert. For traction forces higher than these values, the deformation of the matrix is very high, and the cell culture looses its network structure.

For high cell traction and low cell-cell adhesion [e.g., Fig. 2 B (right, i)], cells align collectively with one another. For these values the cells are polarized but do not form networks: they form lattices, since they are too dispersed.

These behaviors are highly dependent on cell density. Effectively, by preforming the same simulations for $N=300$ cells [Fig. 3 (left)], networks emerge in only a very narrow interval of cell traction force. This is the region of parameters for which the cells are better polarized, so they are able to reach each other and link into a network. Therefore, while Figs. 3 (right, a) and 3 (right, b) have roughly the same characteristics as before, Figs. 3 (right, d) and 3 (right, g) no longer assemble networks since there are not enough cells. In Fig. 3 (right, c) cells adhere strongly to form small clusters that are linked to each other through polarized cells.

For 300 cells, the transition between clusters and dispersed cells appears to be smoother. However, at higher adhesions, the low cell density in this simulation forces some of these clusters to have very few cells, which leads to morphology of clusters mixed with dispersed cells, as is observed in Fig. 3 (right, e). Figure 3 (right, h) still displays high variability of cell shapes, although they no longer align in network-like structures. Also, at these low cell densities it is harder to obtain the collective alignment observed previously for low adhesion and higher traction forces [see Fig. 3 (right, i)].

\section{B. Emerging network morphological data}

When performing the connected component analysis of the medium, we gathered the information about the average number of meshes in a network and their average size. Since these quantities make sense only in the presence of networks, we look into the data only for the morphologies with a fraction of network cells above 0.5, i.e., in the blue region of Figs. 2 (left) and 3 (left).

For values of $\mu \geqslant 1.0 \mathrm{Nm}^{-1}$, the average number of meshes for both $N=300$ cells [Fig. 4(a)] and $N=500$ cells [Fig. 4(b)], decreases as cell-cell adhesion increases, since the branches are thicker, as we saw above. This behavior is accompanied by an increase in average network mesh size (Fig. 5).

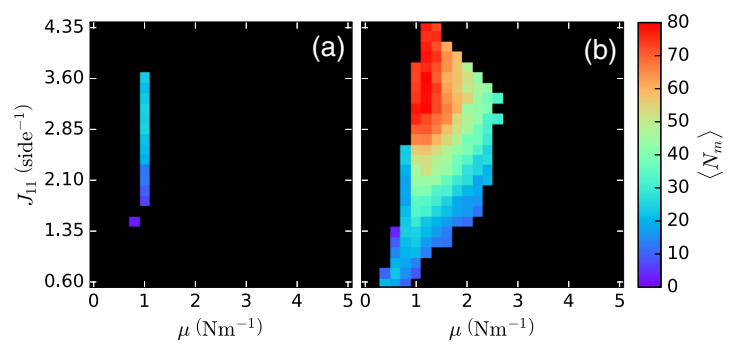

FIG. 4. Average number of meshes out of (a) 300 and (b) 500 cells in a $300 \times 300$ pixel grid with rigidity $E_{0}=10 \mathrm{kPa}$, as a function of traction force, $\mu$, and cell-cell adhesion cost, $J_{11}$. Each data point represents the average of 10 simulations. Because this result is relevant only in cases where networks emerge, only instances where the fraction of networks is above 0.5 are represented. (a) Due to the low cell concentration, the interval where networks emerge is very narrow. The general trend is that decreasing cell-cell adhesion increases average mesh number. (b) Below $\mu=1$, the number of meshes remains low; on the other hand, for values equal or larger than $\mu=1$, the average number of meshes increases as the cell-cell adhesion decreases, as a general trend.

These results suggest that the cell traction force is mainly responsible for the existence or nonexistence of a network structure, and cell-cell adhesion regulates network morphology (number of meshes and their size).

To obtain these results we did not need to describe explicitly the intracellular cytoskeleton rearrangement dynamics, and we assumed that the cells respond immediately to the mechanical microenvironment. Strikingly, the formation of the networks occurs in a larger range of traction forces in the case when the cells take a finite time to respond to their mechanical environment. In fact, in Fig. 6 we compare the previous results with the average cell network fraction as a function of the cell traction force when there is a delay in the cells' response. We observe that the fraction of cells forming a network is similar

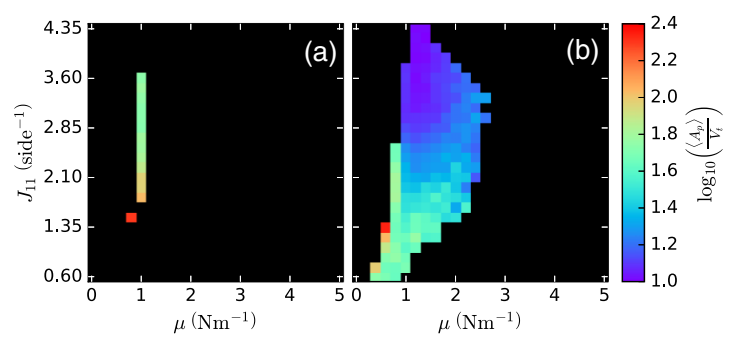

FIG. 5. Logarithm of the average size of patches (divided by the target volume of a cell) obtained from (a) 300 and (b) 500 cells in a $300 \times 300$ pixel grid with rigidity $E_{0}=10 \mathrm{kPa}$, as a function of traction force, $\mu$, and cell-cell adhesion cost, $J_{11}$. Each data point represents the average of 10 simulations. Because this result is relevant only in cases where networks emerge, only instances where the fraction of networks is above 0.5 are represented. (a) Due to the low cell concentration, the interval where networks emerge is very narrow. The general trend is that decreasing cell-cell adhesion also decreases average mesh size. (b) Below $\mu=1 \mathrm{Nm}^{-1}$, the size of meshes remains large; on the other hand, for values equal or larger than $\mu=1 \mathrm{Nm}^{-1}$, the average size of meshes decreases as the cell-cell adhesion decreases. 


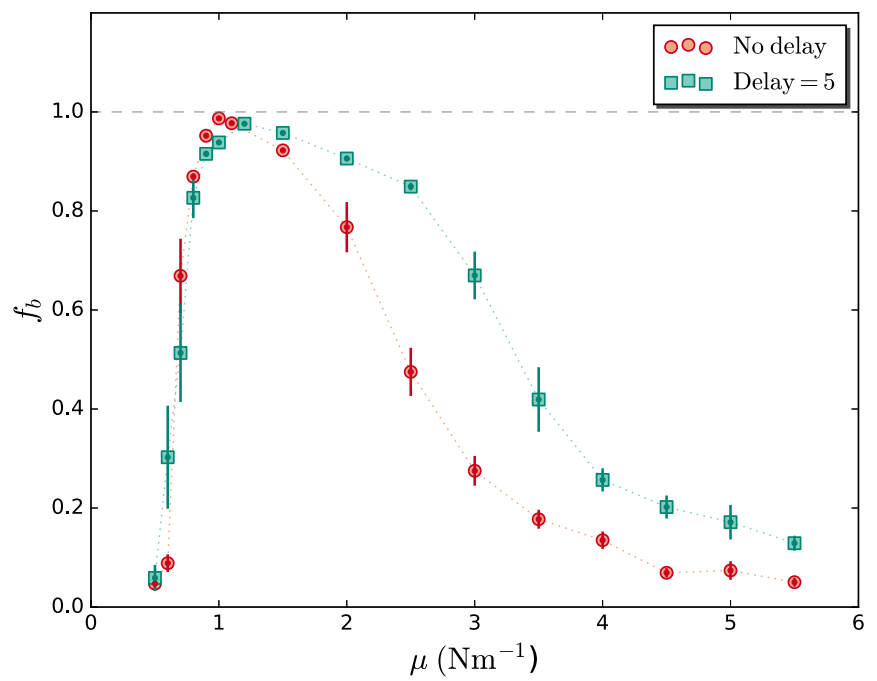

FIG. 6. Average cell network fraction for 500 cells on a $300 \times 300$ pixel grid $\left(0.5625 \mathrm{~mm}^{2}\right)$ with rigidity $E_{0}=10 \mathrm{kPa}$, as a function of traction force, $\mu$, from $\mu=0.5 \mathrm{Nm}^{-1}$ to $\mu=5.5 \mathrm{Nm}^{-1}$ with cell-cell adhesion cost of $J_{11}=2.55$. The green data points correspond to the results obtained when the endothelial cells take five Monte Carlo steps to respond to the mechanical characteristics of their microenvironment. Each point corresponds to the average of 10 simulations.

in both cases, but that the range of traction forces for which the networks are observed is even larger in the case with a small delay in cell response. We also observe that the minimum traction force required for network formation is the same in the case of time delay.

\section{Dependence on ECM stiffness}

We measured the fraction of networks for three different values of stiffnesses, $E_{0}=3,10,14 \mathrm{kPa}$, as a function of the traction force for a cell-cell adhesion cost of $J_{11}=2.55$, for $N=500$ cells (Fig. 7) and $N=300$ cells (Fig. 8). As the stiffness increases, the cell traction force value range where networks emerge gets narrower and shifted towards lower values of $\mu$. Also, for lower number of cells in culture, the range of cell traction forces that lead to network formation is smaller, yet the lower bound of each one of those intervals does not change appreciably with the number of cells in culture.

This critical traction force for network formation, $\mu_{c}$, depends strongly on the ECM's Young's modulus. From Figs. 2 (left), 3 (left), 7 , and 8, for $J_{11}=2.55$, the critical value for $\mu_{c}$ is approximately $\mu_{c}\left(E_{0}=10 \mathrm{kPa}\right) \approx 0.9 \mathrm{Nm}^{-1}$. Also from Figs. 7 and 8 , for $E_{0}=3 \mathrm{kPa}$, the critical value is approximately $\mu_{c}\left(E_{0}=3 \mathrm{kPa}\right) \approx 2.25 \mathrm{Nm}^{-1}$. Last, for $E_{0}=14 \mathrm{kPa}$ the value of $\mu_{c}$ is also approximately the same for $N=500$ and $N=300$ cells, and given by $\mu_{c}\left(E_{0}=14 \mathrm{kPa}\right) \approx 0.40 \mathrm{Nm}^{-1}$.

Since the ECM rigidity is relatively simple to change in an experimental setup, the measurement of the range of Young's modulus for which the networks can be observed as a function of the number of cells in the culture could be used to validate the model, and to infer the model's parameters such as the cell-cell adhesion and the traction force exerted by the cells.

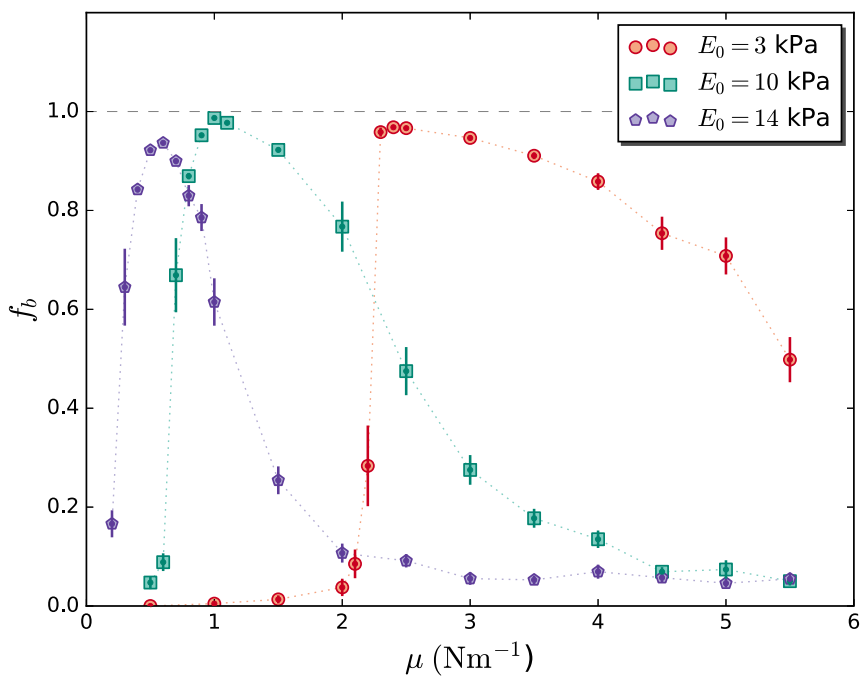

FIG. 7. Average cell network fraction for 500 cells on a $300 \times$ 300 pixel grid $\left(0.5625 \mathrm{~mm}^{2}\right)$ with rigidities $E_{0}=3,10,14 \mathrm{kPa}$, as a function of traction force, $\mu$, from $\mu=0.5 \mathrm{Nm}^{-1}$ to $\mu=6.0 \mathrm{Nm}^{-1}$ with cell-cell adhesion cost of $J_{11}=2.55$. Each point corresponds to the average of 10 simulations.

The quasi-independence of the $\mu_{c}$ on the number of cells in the culture, on the presence of a small delay in the intracellular response, and on the adhesion suggests that we can estimate its value by inspecting the steady-state behavior of a single cell in an elastic matrix. The observation that the cells become strongly polarized at $\mu_{c}$ supports this hypothesis. Therefore we will estimate theoretically the value of $\mu_{c}$.

Consider a rectangular cell in the simulation with sides $l_{x}$ and $l_{y}$ pixels, and with fixed volume $V_{t}=l_{x} l_{y}$. Let $\gamma=\sqrt{l_{x} / l_{y}}$ be a measure of the degree of polarization of the cell. Note that $l_{x}=\sqrt{V_{t}} \gamma$ and $l_{y}=\sqrt{V_{t}} / \gamma$. Without loss of generality, we

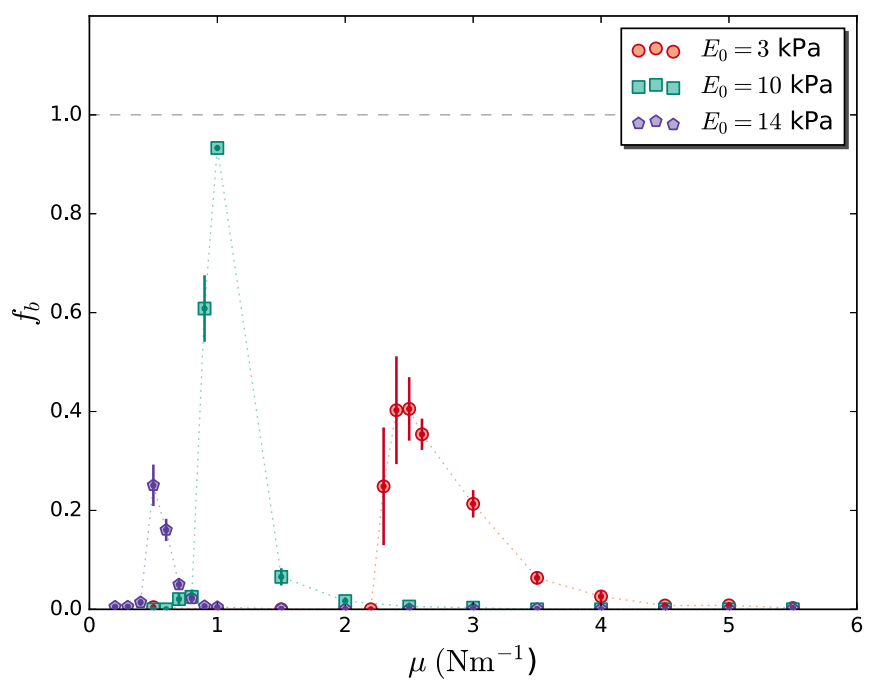

FIG. 8. Average cell network fraction for 300 cells on a $300 \times$ 300 pixel grid $\left(0.5625 \mathrm{~mm}^{2}\right)$ with rigidities $E_{0}=3,10,14 \mathrm{kPa}$, as a function of traction force, $\mu$, from $\mu=0.5 \mathrm{Nm}^{-1}$ to $\mu=6.0 \mathrm{Nm}^{-1}$ with cell-cell adhesion cost of $J_{11}=2.55$. Each point corresponds to the average of 10 simulations. 
will consider that the cell is polarized along the $x$ axis, such that a small increase in $\gamma$ implies a retraction along the $y$ axis and an extension along the $x$ axis. Conversely, a small decrease in $\gamma$ implies a retraction along the $x$ axis and an extension along the $y$ axis. We will look into the effect of the durotaxis term (4) of the Hamiltonian, and we will see for which traction force it will be energetically more favorable for the cells in the matrix to acquire a value of $\gamma$ larger than the unity.

Given a cell with shape defined by the parameter $\gamma$ aligned in the $x$ direction, the number of lattice sites during a MHA step that are able to be added to the cell along the $x$ axis and along the $y$ axis are proportional to $l_{y}$ and $l_{x}$, respectively. The acceptance probability of increasing $\gamma$ can then be approximated to the probability of an expansion along $x$ axis after having retracted along the $y$ axis, i.e., $p(\gamma+\delta \gamma \mid \gamma)=p_{x}^{e}(\gamma) p_{y}^{r}(\gamma)$. Likewise, a decrease in $\gamma$ is approximately $p(\gamma-\delta \gamma \mid \gamma)=p_{y}^{e}(\gamma) p_{x}^{r}(\gamma)$. However, since the durotaxis term in the Hamiltonian is negative for extensions, $p_{x}^{e}(\gamma)=p_{y}^{e}(\gamma)=1$. Considering that the two states of the cell with $\gamma$ and $\gamma+\delta \gamma$ have approximately the same probabilities of retraction, i.e., $p_{x}^{r}(\gamma) \approx p_{x}^{r}(\gamma+\delta \gamma)$ and $p_{y}^{r}(\gamma) \approx p_{y}^{r}(\gamma+\delta \gamma)$, the equation for equilibrium between both states becomes

$$
l_{y} p_{y}^{r}(\gamma)=l_{x} p_{x}^{r}(\gamma)
$$

where the left-hand side represents the probability of increasing $\gamma$ weighted by the elements that are able to extend along the $x$ axis and, in a similar manner, the right-hand side represents probability for decreasing $\gamma$. In equilibrium these two probabilities are identical. Let $\chi=l_{y} p_{y}^{r}(\gamma)-l_{x} p_{x}^{r}(\gamma)$, such that when $\chi>0$ the cell tends to become more polarized (in the $x$ direction), and when $\chi<0$, the cell dynamics tends to decrease $\gamma$.

In order to calculate the probabilities of retraction, we will start from looking into the forces acting on the ECM by the cell. The traction force field of that cell is given by the continuum version of the Lemmon and Romer traction force model (2) applied to this particular cell:

$$
\boldsymbol{f}(\boldsymbol{x})=\int_{-l_{x} / 2}^{l_{x} / 2} \int_{-l_{y} / 2}^{l_{y} / 2} d x^{\prime} d y^{\prime} \mu\left(\boldsymbol{x}^{\prime}-\boldsymbol{x}\right)=-\mu V_{t} \boldsymbol{x} .
$$

Note that $\mu$ is corresponds to the traction force parameter per pixel in the simulation $\left(V_{t}\right.$ is the area of the cell in number of pixels), and it is a scaled parameter already accounting for ECM thickness, meaning that the force $\boldsymbol{f}(\boldsymbol{x})=-\mu V_{t} \boldsymbol{x}$ has units of stress. By using the definition of the Young's modulus, the strain in the matrix can then be estimated by

$$
\begin{aligned}
& \varepsilon_{x x}\left(l_{x} / 2,0\right) \approx \frac{\mu V_{t} l_{x}}{2 E_{0}}, \\
& \varepsilon_{y y}\left(0, l_{y} / 2\right) \approx \frac{\mu V_{t} l_{y}}{2 E_{0}} .
\end{aligned}
$$

Calculating the alteration of the Young's modulus using the strain-stiffening approximation (3) yields

$$
\begin{aligned}
& E_{x}(\gamma)=E_{0}+\rho \mu \gamma, \\
& E_{y}(\gamma)=E_{0}+\frac{\rho \mu}{\gamma},
\end{aligned}
$$

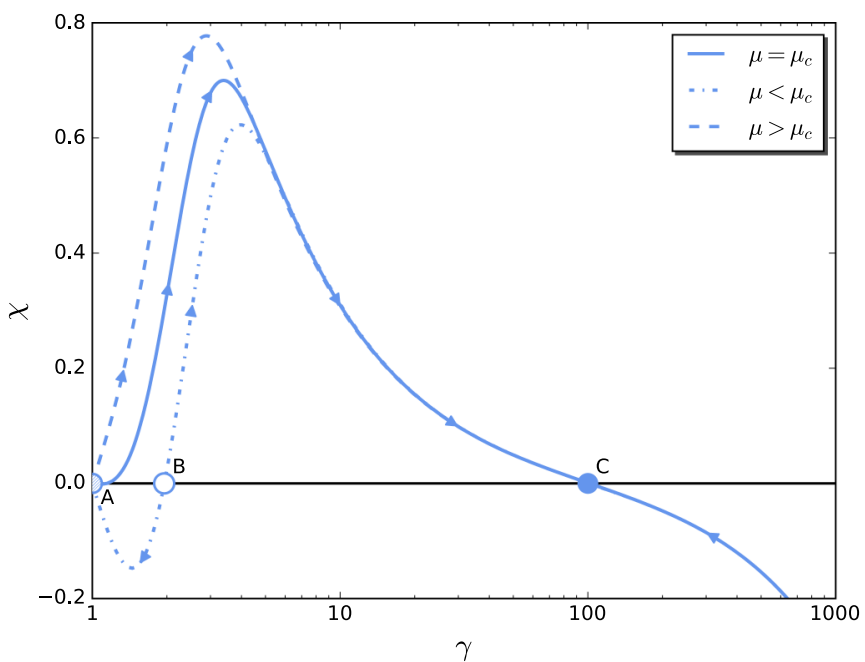

FIG. 9. Representation of the function $\chi$, defined in Eq. (14), for $E_{0}=10 \mathrm{KPa}$, as a function of the anisotropy parameter $\gamma$, for three different values of $\mu$. When $\mu<\mu_{c}$, there are two stable fixed points, A and C, and one unstable fixed point, B. Since A is stable, near isotropic cells prefer to remain apolar. As $\mu$ increases, the unstable point $\mathrm{B}$ moves towards fixed point $\mathrm{A}$ until, at $\mu=\mu_{c}$, B ceases to exist for $\mu>0$ and $\mathrm{A}$ becomes unstable. For values of $\mu>\mu_{c}$ cells prefer to polarize instead of becoming isotropic. Furthermore, it is readily apparent that the value of the derivative of $\chi$ at $\gamma=1$ dictates whether $\mathrm{A}$ is stable or unstable. The values of $\gamma$ at the $\mathrm{C}$ stable point are overestimated due to not taking into account the interface energy term of the CPM.

where $E_{x}$ is the perceived Young's modulus along the $x$ axis of the cell surface at $x=l_{x} / 2, E_{y}$ is the perceived Young's modulus along the $y$ axis of the cell surface at $y=l_{y} / 2$, and $\rho=V_{t}^{3 / 2} /\left(2 \epsilon_{0}\right)$. The durotaxis term of each retraction, where it is assumed all motion is performed along the eigenvectors of strain, is simply given by $f\left(E_{x}(\gamma)\right)$ and $f\left(E_{y}(\gamma)\right)$, using Eq. (5). Finally, the probabilities of retraction are given by

$$
\begin{aligned}
& p_{x}^{r}(\gamma)=e^{-\frac{\alpha}{1+e^{-\beta\left(E_{0}-E_{1 / 2}+\rho \mu \gamma\right)}},} \\
& p_{y}^{r}(\gamma)=e^{-\frac{\alpha}{1+e^{-\beta\left(E_{0}-E_{1 / 2}+\rho \mu / \gamma\right)}},}
\end{aligned}
$$

and $\chi$ is

$$
\chi(\gamma)=\frac{\sqrt{V_{t}}}{\gamma} e^{-\frac{\alpha}{1+\zeta e^{-\beta \rho \mu / \gamma}}}-\sqrt{V_{t}} \gamma e^{-\frac{\alpha}{1+\zeta e^{-\beta \rho \mu \nu}}},
$$

where $\zeta=e^{-\beta\left(E_{0}-E_{1 / 2}\right)}$.

By plotting $\chi$ as a function of $\gamma$ for several values of $\mu$ (see Fig. 9 for an example plotted for $E_{0}=10 \mathrm{KPa}$ ), it is possible to observe a bifurcation of the nonpolarized configuration $(\gamma=$ 1). In fact, this configuration is stable for $\mu$ below a critical value $\mu_{c}$, and it becomes an unstable fixed point for $\mu>\mu_{c}$. Strikingly, increasing the traction force even further brings the polarized cell stable fixed point to lower values of $\gamma$, until again there is only a single fixed point at $\gamma=1$.

From Fig. 9 it becomes readily apparent that when $\mu$ reaches its critical value,

$$
\mu=\left.\mu_{c} \Rightarrow \frac{\partial \chi(\gamma)}{\partial \gamma}\right|_{\gamma=1}=0 .
$$




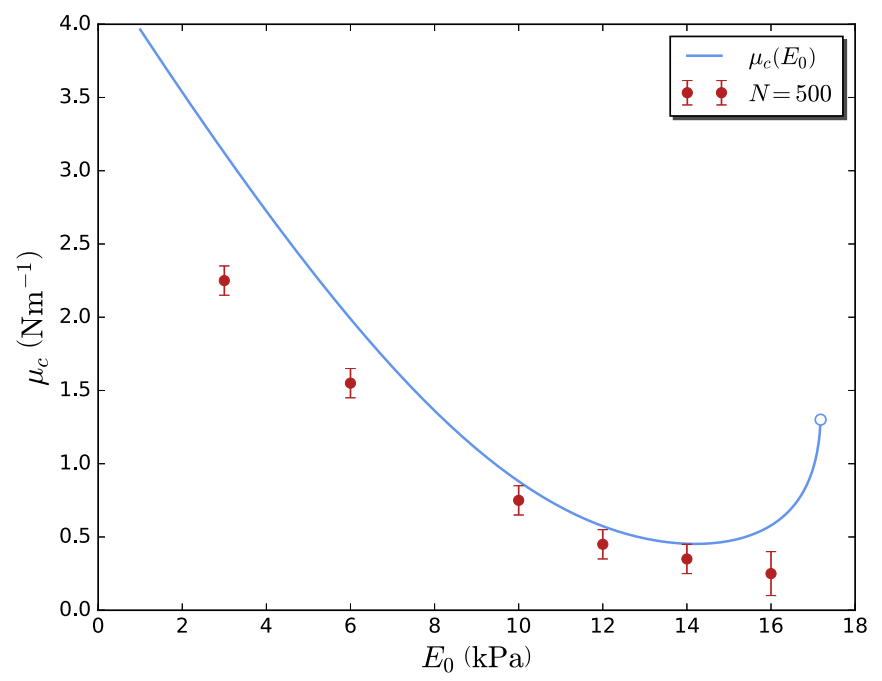

FIG. 10. Critical value of the traction force parameter, $\mu_{c}$, as a function of substrate stiffness, $E_{0}$. Comparison between the theoretical prediction (16), blue line, and estimated values of $\mu_{c}$ from simulated results for $E_{0}=3,6,10,12,14,16 \mathrm{kPa}$, for $N=500$ cells. The prediction for mid- to high values of $E_{0}$ is fairly accurate, considering the precision of the estimative and the approximations made when deriving Eq. (16). The theoretical prediction of $\mu_{c}\left(E_{0}\right)$ is obtained by numerically searching for the smallest root of the left-hand side of Eq. (16) for a fixed value of $E_{0}$.

By plugging $\chi(\gamma)$ from Eq. (14) into this condition for criticality, we obtain the equation that $\mu_{c}$ must obey

$$
\cosh \left[\beta\left(E_{0}-E_{1 / 2}+\rho \mu_{c}\right)\right]-\frac{\alpha \beta \rho}{2} \mu_{c}+1=0 .
$$

This equation can have up to two solutions, depending on the value of $E_{0}$. When $E_{0}$ is large, the durotaxis signal is saturated according to Eq. (5), and there is no solution to this equation. For the parameters $\alpha, \beta, E_{1 / 2}, V_{t}$, and $\epsilon_{0}$ used in this work, the maximum value $E_{0}$ can take and there still exist a polarized stable fixed point is $E_{0}^{\max } \approx 17.2 \mathrm{kPa}$. Below that value for the Young's modulus, there are two solutions to the above equation, with the transition from the unpolarized to polarized cell being the one with smaller $\mu_{c}$. Solving Eq. (16) numerically and obtaining $\mu_{c}$ as a function of $E_{0}$ (Fig. 10) permits a comparison with the values of $\mu_{c}$ obtained from our simulation results.

For such a simple prediction, supposing the cell a rectangle and not considering the influence of cell-cell adhesion, the agreement with the simulation works rather well, especially for mid- to high values of ECM stiffness. This study indicates that there is a critical traction force in the simulation that is able to polarize the cells. When one cell becomes polarized, it is able to exert more force in the matrix, deforming it more, and guiding the neighboring cells to align with it. In this way the networks are formed.

\section{CONCLUSION}

The formation of a functional capillary network is fundamental in many biological processes, from embryogenesis to wound healing. This complex process involves many factors, including endothelial cell adhesion and migration on the ECM. We have shown, using a CPM connected with the FEM, that if cell-cell communication and alignment in these 2D systems is mainly driven by mechanical interactions, then the formation of networks require the polarization and alignment of the endothelial cells. This alignment occurs in substrates of suitable rigidity that can be deformed by the cell's traction forces. The cell can then use the substrate's strain-stiffening property to become polarized. This implies the existence of a minimum value of traction force required for the formation of networks. This critical traction force is independent of the cell number or of cell-cell adhesion. We have estimated analytically the value for the critical traction force, and our estimative agreed quite well with the simulation results. Moreover, cell-cell adhesion is responsible for controlling how the cells group themselves, either as dispersed cells, or as clusters. If the cells form networks, cell-cell adhesion is then important to determine the network morphology (number and size of meshes). This can then be tested experimentally, for example, by observingin vitro the resulting patterns formed by endothelial cells that have different expression levels of adhesion molecules such as VE cadherins.

Though these mechanical interactions have been shown to be very important $[29,30,42-45,49,50]$ other factors, such as chemical gradients, may also play a role in network formation in 2D substrates. Testing experimentally the predictions of the model also tests the role that mechanics plays in pattern formation. In particular, the forces exerted by cells on the matrix could be measured in ECMs of different rigidities and correlated with the cells' capacity for polarization, motility, and the observed pattern morphology. Moreover, the method that we use to extract quantitative data from simulations (fraction of cells in a network, dispersed or clustered) provides a quantitative and reliable way to classify the morphologies observed. This method can also be used to characterize the morphologies observed in in vitro cell cultures.

\section{ACKNOWLEDGMENTS}

The authors acknowledge the support of FEDER funds through the Operational Programme Competitiveness Factors (COMPETE) and of the Foundation for Science and Technology (FCT) through the strategic project UID/FIS/04564/2016. The simulation runs were carried out at the Navigator computer cluster at the University of Coimbra. The simulations were carried out in the Navigator Computer Cluster. The authors thank Roeland Merks for fruitful discussions.

\section{APPENDIX: FINITE ELEMENT METHOD (FEM)}

The FEM reduces a difficult partial differential equation problem into a large number of simpler equations solved locally, eliminating all spatial derivatives in the process. The domain is divided into smaller subdomains, called elements, which are polygons whose vertices are called nodes. For a steady state solution, this method results in a set of algebraic equations that relate the nodes of adjacent elements. These equations are solvable by error minimization. 
For our system, it is advantageous to divide the modeled domain into a grid of square elements with the same length, $L$, overlaying the CPM pixel grid.

The resulting system of equations can be written as

$$
K \boldsymbol{q}=\boldsymbol{f},
$$

where $K$ is the stiffness matrix, $\boldsymbol{q}$ is a vector containing the displacements of all nodes, and $f$ is a vector containing the forces applied to all nodes. Note that, since the displacements and forces are vectorial quantities, $\boldsymbol{q}$ and $\boldsymbol{f}$ store both components in a predetermined pattern. The matrix $K$ is assembled from smaller matrices that relate the displacements to the forces on a single element. Since the elements' shape and size are not altered during the simulation, the local stiffness matrix, $K^{(e)}$, needs to be computed only once.

The local set of equations is obtained by applying the principle of virtual work. Consider a displacement field, $\boldsymbol{u}^{(e)}$, defined inside the element by interpolating the displacement values at the nodes, $\boldsymbol{q}^{(e)}$. The matrix $K^{(e)}$ of the resulting system of equations is given by

$$
K^{(e)}=\int_{-1}^{1} \int_{-1}^{1} \frac{L^{2}}{4} B^{T} C B d \xi d \eta,
$$

where the integral is performed over the volume (area in this case) of the element, with local coordinates $\xi$ and $\eta$, $B=B(\xi, \eta)$ is a matrix that relates the node displace- ment components to the strain tensor in vector form, $\boldsymbol{\varepsilon}=$ $\left(\varepsilon_{x x}, \varepsilon_{y y}, 2 \varepsilon_{x y}\right)=\boldsymbol{\varepsilon}(\xi, \eta)$,

$$
\boldsymbol{\varepsilon}(\xi, \eta)=B(\xi, \eta) \boldsymbol{q}^{(e)},
$$

and $C$ is the stiffness tensor of the constitutive equation for an homogeneous isotropic elastic material,

$$
\sigma(\xi, \eta)=C \varepsilon(\xi, \eta),
$$

where $\sigma=\left(\sigma_{x x}, \sigma_{y y}, \sigma_{x y}\right)=\sigma(\xi, \eta)$ is the stress tensor in vector form. The ECM is considered to be under plane stress conditions, hence $C$ depends only on the Young's modulus, $E_{0}$, and on the Poisson's ratio, $v$,

$$
C=\frac{E_{0}}{1-v^{2}}\left(\begin{array}{ccc}
1 & v & 0 \\
v & 1 & 0 \\
0 & 0 & \frac{1}{2}(1-v)
\end{array}\right)
$$

The double integral is computed using the Gaussian quadrature method for $n=2$ twice. The global stiffness matrix is assembled from the local matrices using the direct stiffness method, which consists in simply adding the components of the local stiffness matrices after correctly mapping local variables into the global ones.

Here, after assembling the matrix $K$, by providing a force vector, displacements are then computed using a preconditioned conjugate gradient method.
[1] P. Carmeliet, Angiogenesis in health and disease, Nat. Med. 9, 653 (2003).

[2] J. Folkman, Angiogenesis in cancer, vascular, rheumatoid and other disease, Nat. Med. 1, 27 (1995).

[3] P. Carmeliet and R. K. Jain, Angiogenesis in cancer and other diseases, Nature (London) 407, 249 (2000).

[4] T. W. Secomb, J. P. Alberding, R. Hsu, M. W. Dewhirst, and A. R. Pries, Angiogenesis: An adaptive dynamic biological patterning problem, PLoS Comput. Biol. 9, e1002983 (2013).

[5] J. Flores, A. M. Romero, R. D. Travasso, and E. C. Poiré, Flow and anastomosis in vascular networks, J. Theor. Biol. 317, 257 (2013).

[6] S. Gloekler and C. Seiler, Natural bypasses can save lives, Circulation 116, e340 (2007).

[7] A. M. T. Rojas, R. Travasso, I. Pagonabarraga, and E. C. Poiré, When do redundant fluidic networks outperform non-redundant ones? Europhys. Lett. 117, 64002 (2017).

[8] M. Kralickova and V. Vetvicka, Role of angiogenesis in endometriosis, Pathol. Disc. 4, 1 (2016).

[9] T. N. Crawford, I. Alfaro, J. B. Kerrison, E. P. Jablon et al., Diabetic retinopathy and angiogenesis, Curr. Diabetes Rev. 5, 8 (2009).

[10] A. Martin, M. R. Komada, and D. C. Sane, Abnormal angiogenesis in diabetes mellitus, Med. Res. Rev. 23, 117 (2003).

[11] Y. Gandica, T. Schwarz, O. Oliveira, and R. D. Travasso, Hypoxia in vascular networks: A complex system approach to unravel the diabetic paradox, PLoS ONE 9, e113165 (2014).

[12] W. Risau, Mechanisms of angiogenesis, Nature (London) 386, 671 (1997).
[13] M. G. Tonnesen, X. Feng, and R. A. Clark, Angiogenesis in wound healing, J. Invest. Dermatol. Symp. Proc. 5, 40 (2000).

[14] R. Demir, A. Yaba, and B. Huppertz, Vasculogenesis and angiogenesis in the endometrium during menstrual cycle and implantation, Acta Histochem. 112, 203 (2010).

[15] H. Gerhardt et al., Vegf guides angiogenic sprouting utilizing endothelial tip cell filopodia, J. Cell Biol. 161, 1163 (2003).

[16] R. D. M. Travasso, E. Corvera Poiré, M. Castro, J. C. RodríguezManzaneque, and A. Hernández-Machado, Tumor angiogenesis and vascular patterning: A mathematical model, PLoS ONE 6, e19989 (2011).

[17] P. Santos-Oliveira, A. Correia, T. Rodrigues, T. M. RibeiroRodrigues, P. Matafome, J. C. Rodríguez-Manzaneque, R. Seiça, H. Girão, and R. D. M. Travasso, The force at the tip: Modelling tension and proliferation in sprouting angiogenesis, PLoS Comput. Biol. 11, e1004436 (2015).

[18] M. Moreira-Soares, R. Coimbra, L. Rebelo, J. Carvalho, and R. D. M. Travasso, Angiogenic factors produced by hypoxic cells drive anastomoses in sprouting angiogenesis (unpublished).

[19] T. Heck, M.-M. Vaeyens, and H. Van Oosterwyck, Computational models of sprouting angiogenesis and cell migration: Towards multiscale mechanochemical models of angiogenesis, Math. Model. Nat. Phenom. 10, 108 (2015).

[20] M. M. Quinas-Guerra, T. M. Ribeiro-Rodrigues, J. C. RodríguezManzaneque, and R. D. M. Travasso, Understanding the dynamics of tumor angiogenesis: A systems biology approach, in Systems Biology in Cancer Research and Drug Discovery, edited by A. S. Azmi (Springer, Dordrecht, 2012), pp. 197-227. 
[21] R. D. M. Travasso, The mechanics of blood vessel growth, in Vasculogenesis and Angiogenesis: From Embryonic Development to Regenerative Medicine, edited by Dr. Dan Simionescu (InTech, Rijeka, Croatia, 2011).

[22] S. Patan, Vasculogenesis and angiogenesis, in Angiogenesis in Brain Tumors, edited by M. Kirsch and P. M. Black (Springer, Boston, MA, 2004), pp. 3-32.

[23] C. J. Drake, J. E. Hungerford, and C. D. Little, Morphogenesis of the first blood vessels, Ann. NY Acad. Sci. 857, 155 (1998).

[24] H. Masuda and T. Asahara, Post-natal endothelial progenitor cells for neovascularization in tissue regeneration, Cardiovasc. Res. 58, 390 (2003).

[25] A. L. George, P. Bangalore-Prakash, S. Rajoria, R. Suriano, A. Shanmugam, A. Mittelman, and R. K. Tiwari, Endothelial progenitor cell biology in disease and tissue regeneration, J. Hematol. Oncol. 4, 24 (2011).

[26] E. W. Ades, F. J. Candal, R. A. Swerlick, V. G. George, S. Summers, D. C. Bosse, and T. J. Lawley, Hmec-1: Establishment of an immortalized human microvascular endothelial cell line, J. Invest. Dermatol. 99, 683 (1992).

[27] R. Shao and X. Guo, Human microvascular endothelial cells immortalized with human telomerase catalytic protein: A model for the study of in vitro angiogenesis, Biochem. Biophys. Res. Commun. 321, 788 (2004).

[28] S. Guo, J. Lok, Y. Liu, K. Hayakawa, W. Leung, C. Xing, X. Ji, and E. H. Lo, Assays to examine endothelial cell migration, tube formation, and gene expression profiles, in Cerebral Angiogenesis, Methods in Molecular Biology, edited by R. Milner (Humana Press, New York, NY, 2014), Vol. 1135.

[29] G. K. Xu, B. Li, X. Q. Feng, and H. Gao, A tensegrity model of cell reorientation on cyclically stretched substrates, Biophys. J. 111, 1478 (2016).

[30] G. K. Xu, C. Yang, J. Du, and X. Q. Feng, Integrin activation and internalization mediated by extracellular matrix elasticity: A biomechanical model, J. Biomechanics 47, 1479 (2014).

[31] R. A. Francescone III, M. Faibish, and R. Shao, A matrigel-based tube formation assay to assess the vasculogenic activity of tumor cells, J. Visual. Exp. 55, 3040 (2011).

[32] D. Manoussaki, S. Lubkin, R. Vemon, and J. Murray, A mechanical model for the formation of vascular networks in vitro, Acta Biotheor. 44, 271 (1996).

[33] J. D. Murray, D. Manoussaki, S. R. Lubkin, and R. Vernon, A mechanical theory of in vitro vascular network formation, in Vascular Morphogenesis: In Vivo, in Vitro, in Mente, Cardiovascular Molecular Morphogenesis, edited by C. D. Little, V. Mironov, and E. H. Sage (Birkhäuser, Boston, 1996), pp. 173-188.

[34] G. Serini, D. Ambrosi, E. Giraudo, A. Gamba, L. Preziosi, and F. Bussolino, Modeling the early stages of vascular network assembly, EMBO J. 22, 1771 (2003).

[35] D. Ambrosi, A. Gamba, and G. Serini, Cell directional and chemotaxis in vascular morphogenesis, Bull. Math. Biol. 66, 1851 (2004).

[36] R. M. Merks, S. V. Brodsky, M. S. Goligorksy, S. A. Newman, and J. A. Glazier, Cell elongation is key to in silico replication of in vitro vasculogenesis and subsequent remodeling, Dev. Biol. 289, 44 (2006).

[37] A. Szabo, E. D. Perryn, and A. Czirok, Network Formation of Tissue Cells Via Preferential Attraction to Elongated Structures, Phys. Rev. Lett. 98, 038102 (2007).

[38] R. M. Merks, E. D. Perryn, A. Shirinifard, and J. A. Glazier, Contact-inhibited chemotaxis in de novo and sprouting bloodvessel growth, PLoS Comput. Biol. 4, e1000163 (2008).

[39] A. Köhn-Luque, W. De Back, J. Starruß, A. Mattiotti, A. Deutsch, J. M. Pérez-Pomares, and M. A. Herrero, Early embryonic vascular patterning by matrix-mediated paracrine signaling: A mathematical model study, PLoS ONE 6, e24175 (2011).

[40] N. Kleinstreuer, D. Dix, M. Rountree, N. Baker, N. Sipes, D. Reif, R. Spencer, and T. Knudsen, A computational model predicting disruption of blood vessel development, PLoS Comput. Biol. 9, e1002996 (2013).

[41] J. Singh, F. Hussain, and P. Decuzzi, Role of differential adhesion in cell cluster evolution: From vasculogenesis to cancer metastasis, Comput. Methods Biomechanics Biomed. Eng. 18, 282 (2015).

[42] C. A. Reinhart-King, M. Dembo, and D. A. Hammer, Cellcell mechanical communication through compliant substrates, Biophys. J. 95, 6044 (2008).

[43] J. P. Califano and C. A. Reinhart-King, A balance of substrate mechanics and matrix chemistry regulates endothelial cell network assembly, Cell. Mol. Bioeng. 1, 122 (2008).

[44] J. P. Califano and C. A. Reinhart-King, Exogenous and endogenous force regulation of endothelial cell behavior, J. Biomechanics 43, 79 (2010).

[45] J. P. Califano and C. A. Reinhart-King, Substrate stiffness and cell area predict cellular traction stresses in single cells and cells in contact, Cell. Mol. Bioeng. 3, 68 (2010).

[46] R. F. M. van Oers, E. G. Rens, D. J. LaValley, C. A. ReinhartKing, and R. M. H. Merks, Mechanical cell-matrix feedback explains pairwise and collective endothelial cell behavior in vitro, PLoS Comput. Biol. 10, 1 (2014).

[47] G. K. Xu, X. Q. Feng, H. P. Zhao, and B. Li, Theoretical study of the competition between cell-cell and cell-matrix adhesions, Phys. Rev. E 80, 011921 (2009).

[48] G. K. Xu, J. Hu, R. Lipowsky, and T. R. Weikl, Binding constants of membrane-anchored receptors and ligands: A general theory corroborated by Monte Carlo simulations, J. Chem. Phys. 143, 243136 (2015).

[49] C. A. Lemmon and L. H. Romer, A predictive model of cell traction forces based on cell geometry, Biophys. J. 99, L78 (2010).

[50] M. S. Hall, F. Alisafaei, E. Ban, X. Feng, C.-Y. Hui, V. B. Shenoy, and M. Wu, Fibrous nonlinear elasticity enables positive mechanical feedback between cells and ECMs, Proc. Natl. Acad. Sci. USA 113, 14043 (2016).

[51] M. L. Gardel, J. H. Shin, F. C. MacKintosh, L. Mahadevan, P. Matsudaira, and D. A. Weitz, Elastic behavior of cross-linked and bundled actin networks, Science 304, 1301 (2016). 